\title{
Planejamento digital para cirurgia guiada com implantes dentários: relato de caso
}

\author{
Digital planning for guided surgery with dental implants: case report \\ Planificación digital para cirugía guiada con implantes dentales: relato de caso
}

Recebido: 09/11/2021 | Revisado: 16/11/2021 | Aceito: 19/11/2021 | Publicado: 29/11/2021

\author{
Ericles Marinho Brito \\ ORCID: https://orcid.org/0000-0001-8435-0267 \\ Faculdade Independente do Nordeste, Brasil \\ E-mail: ericlesmarinho.odonto@gmail.com \\ Tyago Souza dos Santos Silva \\ ORCID: https://orcid.org/0000-0001-7702-6426 \\ IMMERSE-Cursos de pós-graduação em odontologia, Brasil \\ E-mail: tyago_silva@hotmail.com \\ Wander Rocha Carvalho \\ ORCID: https://orcid.org/0000-0001-7318-1015 \\ IMMERSE-Cursos de pós-graduação em odontologia, Brasil \\ E-mail: drwanderrocha@gmail.com \\ Ricardo Silva Alves \\ ORCID: https://orcid.org/0000-0002-2444-260X \\ Faculdade Independente do Nordeste, Brasil \\ E-mail: alvesricardodr@gmail.com
}

\begin{abstract}
Resumo
Introdução: Um dos maiores desafios das cirurgias com implantes dentários, hoje em dia, é a posição ideal em que os implantes serão instalados, ou seja, a posição tridimensional. A inclinação e o paralelismo em relação aos dentes adjacentes e demais implantes devem ser respeitadas a fim de obter uma melhor distribuição de força, evitando assim fraturas, fadigas e reabsorções ósseas, além de preservar estruturas anatômicas nobres. Entretanto, na atualidade, a odontologia vem se tornando mais digital e já pode contar com equipamentos tecnológicos, exames tomográficos e scanners, que dão a possibilidade de planejar todo o processo cirúrgico através de softwares, além de possibilitar a confecção de guias cirúrgicos, através de impressoras 3D ou fresadoras, que irão orientar a posição tridimensional do implante. Objetivo: Demonstrar a importância do processo de planejamento digital em cirurgia de implante, enfatizando as técnicas de cirurgia guiada na implantodontia, através de um relato de caso. Descrição do caso: Paciente do gênero masculino, 58 anos, ASA I, leucoderma, compareceu a Clínica Escola do Centro de Odontologia Contemporânea Upgrade, durante a Especialização de Periodontia e Implantodontia, relatando uma insatisfação com seu sorriso, devido diversas perdas dentárias. Ao exame clínico, observou a presença de doença periodontal em todos os elementos remanescentes e mordida cruzada anterior. Dessa forma, optou-se pela exodontia dos elementos dentários e a confecção de próteses totais sobre implante, com planejamento virtual, utilizando guia cirúrgico planejado e específico para cirurgia guiada.
\end{abstract}

Palavras-chave: Implantodontia; Tomografia computadorizada; Cirurgia guiada.

\begin{abstract}
Introduction: One of the biggest challenges in dental implant surgeries today is the ideal position in which the implants will be installed, that is, the three-dimensional position. The inclination and parallelism in relation to adjacent teeth and other implants must be respected in order to obtain a better distribution of force, thus avoiding fractures, fatigue and bone resorption, in addition to preserving noble anatomical structures. However, nowadays, dentistry is becoming more digital and can now rely on technological equipment, tomographic exams and scanners, which give the possibility to plan the entire surgical process through software, in addition to enabling the production of surgical guides, through 3D printers or milling machines, which will guide the three-dimensional position of the implant. Objective: To demonstrate the importance of the digital planning process in implant surgery, emphasizing guided surgery techniques in implant dentistry, through a case report. Case description: Male patient, 58 years old, ASA I, leucoderma, attended the School Clinic of the Center for Contemporary Dentistry Upgrade, during the Specialization in Periodontics and Implantology, reporting dissatisfaction with his smile, due to several tooth loss. On clinical examination, he noted the presence of periodontal disease in all remaining elements and anterior crossbite. Thus, we opted for the extraction of dental elements and the manufacture of complete dentures on implants, with virtual planning, using a planned surgical guide specific for guided surgery.
\end{abstract}

Keywords: Implantology; Computed Tomography; Guided surgery. 


\begin{abstract}
Resumen
Introducción: Uno de los mayores retos de las cirugías de implantes dentales en la actualidad es la posición ideal en la que se instalarán los implantes, es decir, la posición tridimensional. Se debe respetar la inclinación y el paralelismo en relación con los dientes adyacentes y otros implantes para obtener una mejor distribución de la fuerza, evitando asî fracturas, fatiga y reabsorción ósea, además de preservar las estructuras anatómicas nobles. Sin embargo, hoy en día la odontología es cada vez más digital y ahora puede contar con equipos tecnológicos, exámenes tomográficos y escáneres, que dan la posibilidad de planificar todo el proceso quirúrgico a través de software, además de posibilitar la producción de guías quirúrgicas, mediante impresoras 3D o fresado. máquinas, que guiarán la posición tridimensional del implante. Objetivo: Demostrar la importancia del proceso de planificación digital en la cirugía de implantes, enfatizando las técnicas de cirugía guiada en implantología, a través de un caso clínico. Descripción del caso: Paciente masculino de 58 años, ASA I, leucoderma, que asistió a la Clínica Escolar del Centro de Actualización de la Odontología Contemporánea, durante la Especialización en Periodoncia e Implantología, refiriendo insatisfacción con su sonrisa, debido a la pérdida de varios dientes. El examen clínico reveló la presencia de enfermedad periodontal en todos los elementos restantes y mordida cruzada anterior. Así, apostamos por la extracción de elementos dentales y la fabricación de prótesis completas sobre implantes, con planificación virtual, utilizando una guía quirúrgica planificada específica para cirugía guiada.
\end{abstract}

Palabras clave: Implantología; Tomografía computarizada; Cirugía guiada.

\title{
1. Introdução
}

A partir das descobertas de Per-Ingvar Branemak, na década de 50, a história da odontologia moderna do implante se iniciou. Branemark descobriu em suas pesquisas que a aposição íntima entre o tecido ósseo e a superfície do implante de titânio seria resistente a transferências de cargas. Esses estudos comprovaram que a deposição de tecido ósseo na superfície dos implantes dependia de relações entre as células e a superfície do implante, o que gerava interações previsíveis e capazes de suportar próteses totais com sucesso à longo prazo. Esse fenômeno é o que conhecemos hoje como "osseointegração" e se tornou o princípio básico para todos os estudos e procedimentos com implantes até nos dias atuais (Carranza et al., 2007).

Atualmente, a grande quantidade de estudos e publicações científicas sobre a osseointegração, comprova que esse tema se tornou bem consolidado e sua compreensão não se apresenta mais como um desafio. Assim, o maior desafio acerca dos procedimentos na implantodontia é a posição em que os implantes serão instalados, o que é chamada de posição 3D (Posição tridimensional). Visto que os primeiros implantes foram instalados tendo como referência somente radiografias e modelos de gesso, a instalação dos implantes requeria tomada de decisões do profissional durante o trascirúrgico, o que demandava bastante conhecimento anatômico e experiência. Além disso, as radiografias fornecem somente a visão da altura do osso e não da espessura, pois é um exame bidimensional, então os procedimentos se tornavam mais sujeitos à erros e falhas (Pereira et al., 2019).

O desenvolvimento de novos exames de imagem, como Tomografia Computadorizada de Feixe de Cônico (CBCT), trouxe consigo a possibilidade do planejamento tridimensional (3D) de cirurgias de implantes, pois fornece informações fidedignas das estruturas anatômicas do paciente, destacando aspectos mais detalhados de estruturas nobres que devem ser levadas em consideração, por exemplo, nervos e vasos que devem ser protegidos, além de um melhor planejamento em relação à posição, paralelismo e inclinação (Jain et al., 2019).

Com o avanço tecnológico e a empregabilidade dessas tecnologias na odontologia, visando a redução de erros no processo de instalação de implantes, e a instalação dos mesmos numa posição precisamente ideal, desenvolveu-se então a cirurgia guiada, que consiste na utilização de dispositivos cirúrgicos que orientarão a posição tridimensional em que será instalado o implante. Estas guias são produzidas com alta precisão e são modelos individualizados para cada paciente, obtidos por meio de softwares e imagens tomográficas (Kraft, 2019).

Hoje em dia existem duas maneiras de se obter um guia cirúrgico, uma delas é a partir do modelo de gesso, obtido pela moldagem com alginato e a outra forma é através de um modelo 3D, com guia fabricado a partir de dados computadorizados que reproduzem o tecido mole e os dentes do paciente, sendo montados pela estereolitografia. Assim, 
simplifica-se a técnica, diminui os erros humanos, aumenta a precisão do local de instalação dos implantes, a previsibilidade do caso e favorece o processo de cicatrização (Kraft, 2019).

Vários programas e softwares estão sendo usados na implantodontia para facilitar o planejamento cirúrgico de implantes dentais. O planejamento virtual consiste em sobrepor imagens adquiridas por meio de tomografias computadorizadas, escaneamentos intraorais e fotografias intra e extrabucais para amplificar a visão diagnóstica, gerando uma estrutura de imagens detalhadas de vários parâmetros anatômicos e estéticos. Esses softwares permitem que o profissional meça a altura, densidade, volume ósseos em um computador pessoal de forma interativa. Tem como principal vantagem a melhor assimilação do tratamento por parte do paciente e para o profissional a possibilidade de visualizar fidedignamente o caso a ser manipulado, aplicando virtualmente o seu planejamento (Veríssimo et al., 2021).

Desde 2002 que vem se aprimorando várias técnicas de cirurgia guiada para implantes dentários, como também ocorre o desenvolvimento de novas tecnologias para de manuseio de imagens, softwares e impressoras 3D. O resultado final do processo de planejamento 3D, que é o guia cirúrgico, oferece várias vantagens ao procedimento, desde incisões minimamente invasivas com alto grau de precisão, angulação dos implantes mais previsíveis, diminuição do tempo clínico e grande estabilidade imediata, devido às fixações e encaixes que já foram anteriormente milimetricamente planejadas (Alvares, 2020).

Portanto, este projeto de pesquisa leva em consideração o novo modelo da odontologia, pois há um relevante aumento do uso de aparelhos e tecnologias na odontologia digital, principalmente em se tratando do planejamento virtual e confecção de guias cirúrgicos por meio de softwares e impressoras 3D.

\section{Metodologia}

O estudo seguiu a tipologia de relato de caso clínico, descritivo e qualitativo (Pereira et al. 2018). Um relato de caso é uma análise e descrição detalhada de uma condição que apresenta alguma característica que a torna notável e colabora para o conhecimento científico. Neste artigo é detalhado um caso de um paciente que foi submetido à cirurgias de instalação de implantes, para reabilitação estética com prótese total sobre implante, utilizando um guia cirúrgico obtido por meio de impressão 3D, tendo como enfoque principal o planejamento digital para a referida cirurgia.

Quanto aos preceitos bioéticos, levou-se em consideração os princípios preconizados pelo Conselho Nacional de Saúde por meio da Resolução Normativa n. 466/2012 e a Carta Circular n. 166/2018. Por se tratar de uma pesquisa que envolve seres humanos, esse trabalho foi aprovado pelo Comitê de Ética em Pesquisa local da Faculdade Independente do Nordeste - FAINOR. O paciente do caso selecionado consentiu e concordou por meio do Termo de Consentimento Livre e Esclarecido (TCLE) em compartilhar suas imagens para uso acadêmico, além do Termo de Autorização de Uso de Imagem e/ou Depoimento.

\section{Relato de Caso}

Paciente do sexo masculino, leucoderma, 58 anos, ASA I, compareceu numa clínica escola de odontologia particular, durante a Especialização de Periodontia e Implantodontia, relatando como queixa principal uma insatisfação com o seu sorriso, devido muitas áreas edêntulas. Após anamnese e exame clínico, foi observado ausências de inúmeros elementos dentários posteriores $(18,17,16,15,14,13,25,26,27,28,38,37,36,35,41,44,45,46,47,48)$ e mordida cruzada anterior, com paciente Classe III, segundo a classificação de Angle (Figura 1). 
Figura 1. Aspecto oral inicial, o qual pode-se observar uma mordida cruzada anterior, o elemento 24 girovertido, inúmeras áreas edêntulas e ausência de suporte ósseo e gengival a nível cervical.

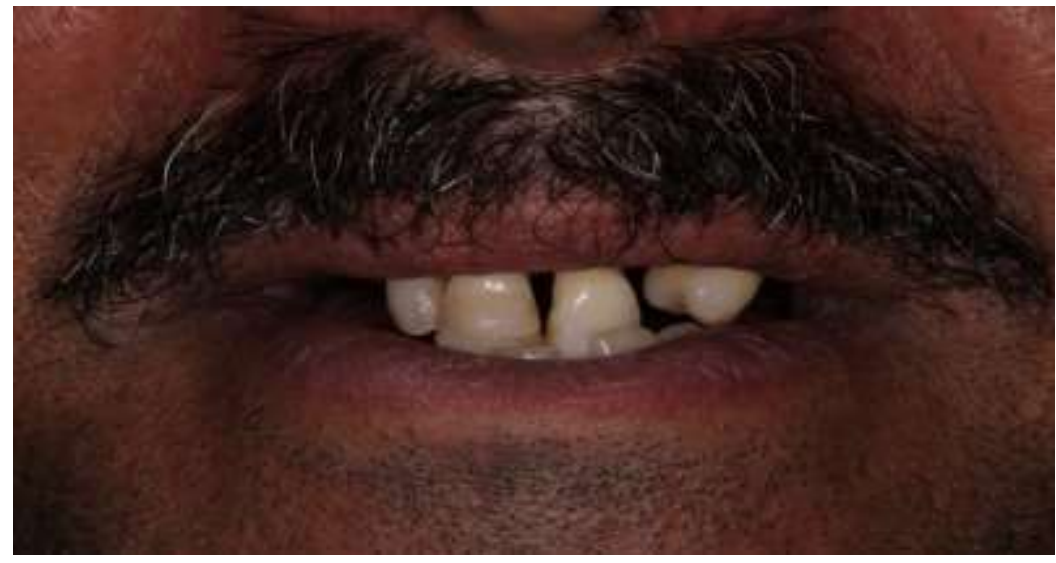

Fonte: Autores.

Além disso, uma grande perda de inserção nos dentes anteriores remanescentes, que após a sondagem, foi diagnosticada como periodontite propriamente dita, estágio IV, grau C, generalizada, segundo a classificação periodontal de Caton et al. 2018. Assim, optou-se pela exodontia dos elementos dentários e confecção de próteses totais sobre implante da arcada superior por meio de planejamento digital e guia cirúrgico e na arcada inferior de maneira convencional, tratamento esse, aceito pelo paciente.

Após a escolha do tratamento, foi solicitado um exame de hemograma completo, para avaliar as condições sanguíneas do paciente, como quantidade de plaquetas, tempo de coagulação e tempo de sangramento. Foi solicitado também o exame de glicemia em jejum, para avaliar o índice de glicose na corrente sanguínea. Esses exames são necessários para o diagnóstico de possíveis patologias que podem tornar o paciente inválido para o procedimento cirúrgico ou exigir do profissional cuidados extras para o transcirúrgico. Solicitou-se também, exames de imagem como radiografia panorâmica e CBCT para auxiliar no planejamento do tratamento.

Depois de avaliar a normalidade dos exames, o tratamento foi iniciado com moldagem dos arcos, com utilização de silicona de adição, leve e pesada, para obtenção do modelo inicial do paciente. Logo depois, fez-se a moldagem periférica para a confecção da prótese total superior imediata. Ainda na mesma sessão, fez-se o plano de cera do paciente para registrar a relação intermaxilar, estabelecer a dimensão vertical de oclusão (DVO) adequada e definir padrões estéticos, como linha do sorriso, corredor bucal e linha média (Figura 2).

Figura 2. Plano de cera para confecção da prótese provisória. Observar marcações que servirão de referência para o plano oclusal, a linha média dental e dimensão vertical de oclusão.

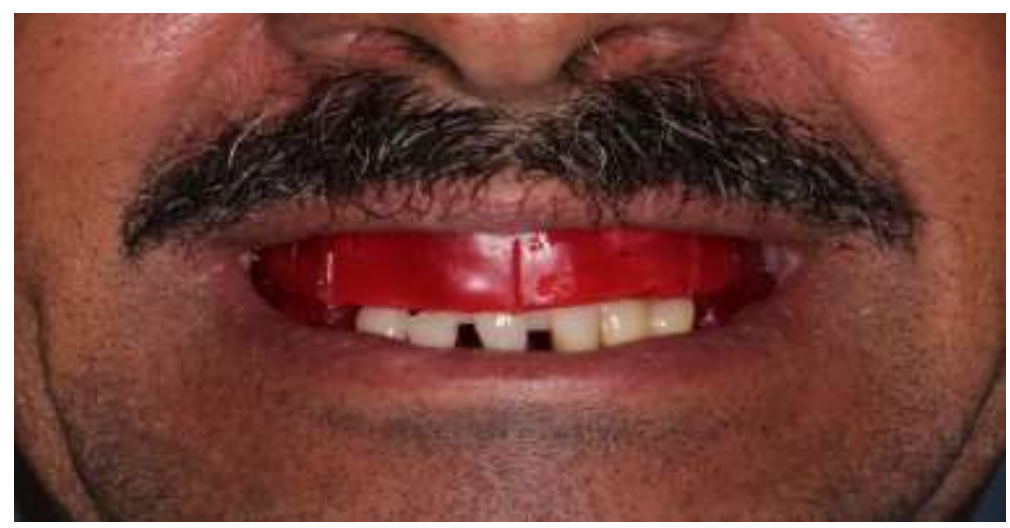

Fonte: Autores. 
$\mathrm{Na}$ sessão posterior, foi realizada as exodontias dos elementos superiores e adaptação imediata da prótese total superior provisória. Após quinze dias de pós cirúrgico, executou-se o reembasamento da prótese total superior. Essa prótese deve ser bem adaptada, pois ela serve como base de referência para o planejamento digital, pois o software utiliza a parte interior da prótese como modelo para uso, afim de registrar a mucosa do paciente. Além disso, é importante para parâmetros oclusais, guiando a posição e relação entre os dentes do arco superior (Figura 3).

Figura 3. Prótese provisória adaptada em boca, a qual pode-se observar todos os parâmetros estéticos e oclusais definidos.

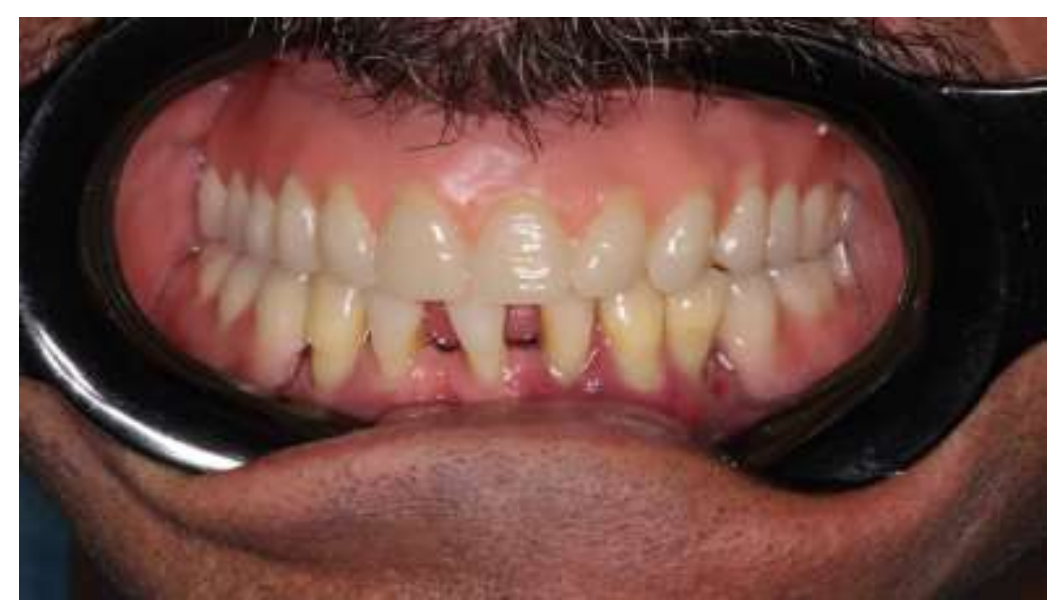

Fonte: Autores.

Foram feitas marcações de guta-percha na prótese total superior provisória, com o intuito de referenciar as relações entre o paciente e a prótese por meio das tomadas tomográficas. Assim, fez duas tomografias, a primeira tomografia foi realizada no paciente utilizando a prótese com as marcações e a segunda somente da prótese. Esses dados tomográficos obtidos foram transferidos para o software de planejamento de implante virtual (Blue Sky Plan, Blue Sky Plan, Estados Unidos), por meio de arquivos de dados de Imagem Digital e Comunicações em Medicina (DICOM), para formular um plano cirúrgico do posicionamento dos implantes (Figura 4). Essas tomografias servem de referência para planejar a posição 3D dos implantes (Arcuri et al., 2015).

Figura 4. Imagem tomográfica da arcada superior com paciente portando prótese com marcações de guta-percha. Pode-se observar as marcações feitas na superfície vestibular da prótese, o que servirá de referência para o planejamento digital.

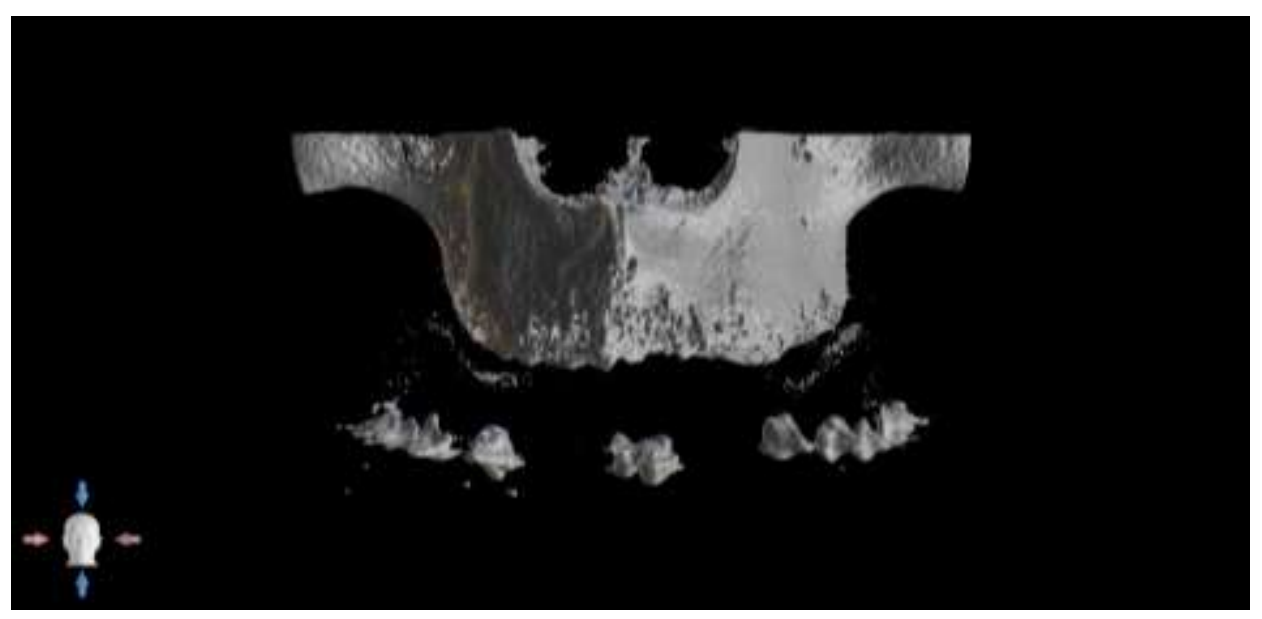

Fonte: Autores. 
Após a coleta de dados, fez se um escaneamento intraoral do paciente, de maneira direta, utilizando o Scanner intraoral (CEREC Omnicam, Dentsply Sirona, Estados Unidos) com o objetivo de gerar um modelo virtual tridimensional que reproduz as estruturas anatômicas com exatidão e precisão, entregando imagens da superfície intraoral, em tamanho real, com proporção de 1:1, com arquivos de estereolitografia (STL) importados para o software responsável por processar esses dados. O scanner é importante no planejamento digital, pois oferece a possibilidade de obtenção de medidas dentais para fins de diagnósticos, estéticos e reabilitações protéticas fidedignas (An et al., 2019).

Com toda a coleta de dados digitais finalizada, foi iniciado o processo de planejamento digital a partir do software específico. Para que se tenha um campo digital de planejamento mais amplo, sobrepôs os dados DICOM aos dados em STL por meio de algoritmos sofisticados que possibilitam essa fusão de arquivos de estereolitografia (STL), gerados a partir de uma varredura ótica 3D obtidos pelo scanner intraoral e dados DICOM adquiridos da CBCT do paciente. Essa fusão é realizada combinando pontos correspondentes nas marcações da prótese (Figura 5). Essa manobra possibilita a visualização do conjunto ósseo do paciente em conjunto com a superfície da mucosa alveolar. Dessa forma, o profissional pode calcular a profundidade da instalação dos implantes, levando em consideração o osso alveolar e a mucosa do paciente. (Cunha et al., 2020).

Figura 5. Imagem tomográfica, na qual pode-se observar a sobreposição de imagens DICOM (cinza) com dados STL (verde) por meio de software.

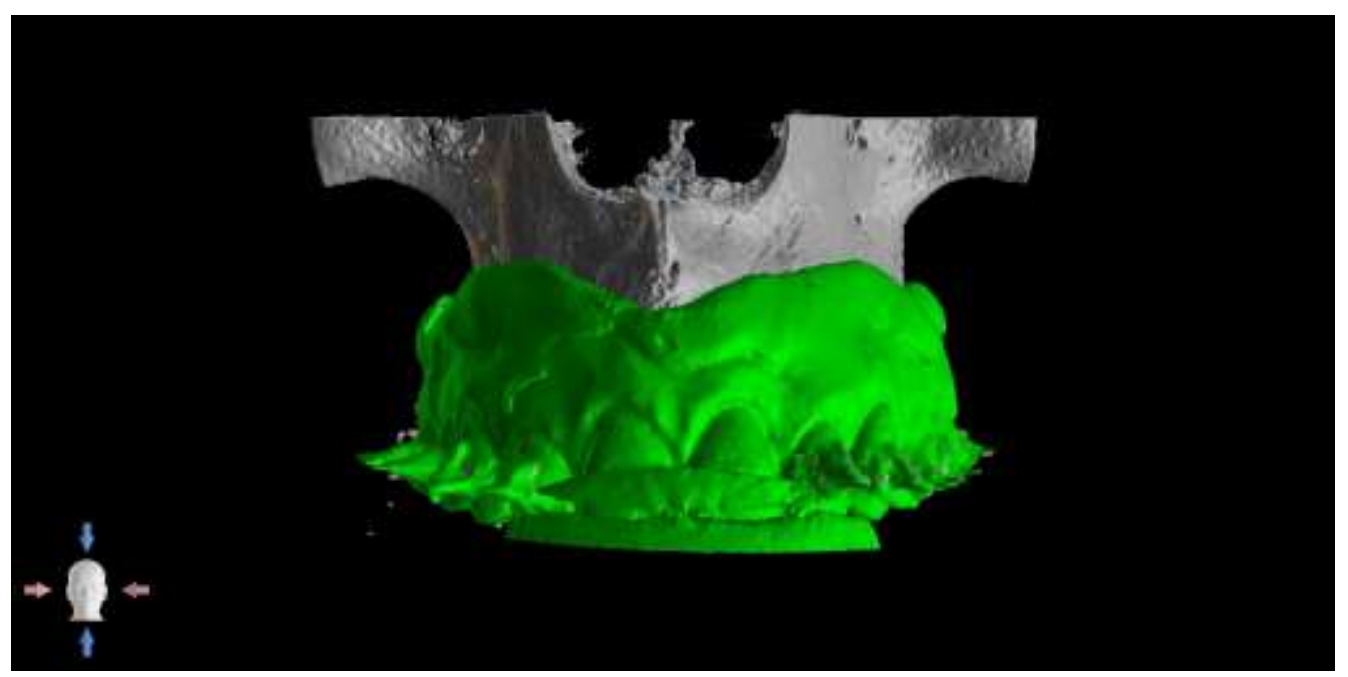

Fonte: Autores.

Utilizando a CBCT da prótese com as marcações, iniciou-se o processo de vazamento digital da mucosa do paciente (Figura 6). Primeiro, o arquivo que se encontrava em formato DICOM, foi convertido em STL, para que se pudesse observar a superfície da mucosa de forma tridimensional e confiável. Após obtenção dessas imagens, o software inverte a base interna da prótese para transformar em um modelo de uso, registrando assim a mucosa por meio digital (Figura 7). Essa imagem foi fundida sobre o campo tomográfico DICOM, tendo como base as marcações de guta percha feitas previamente na prótese. (Cunha et al., 2020). 
Figura 6. Vazamento digital da mucosa do paciente, demonstrando em azul o registro digital da superfície do interior da prótese feito pelo software, que preenche o interior da prótese e gera um modelo virtual.

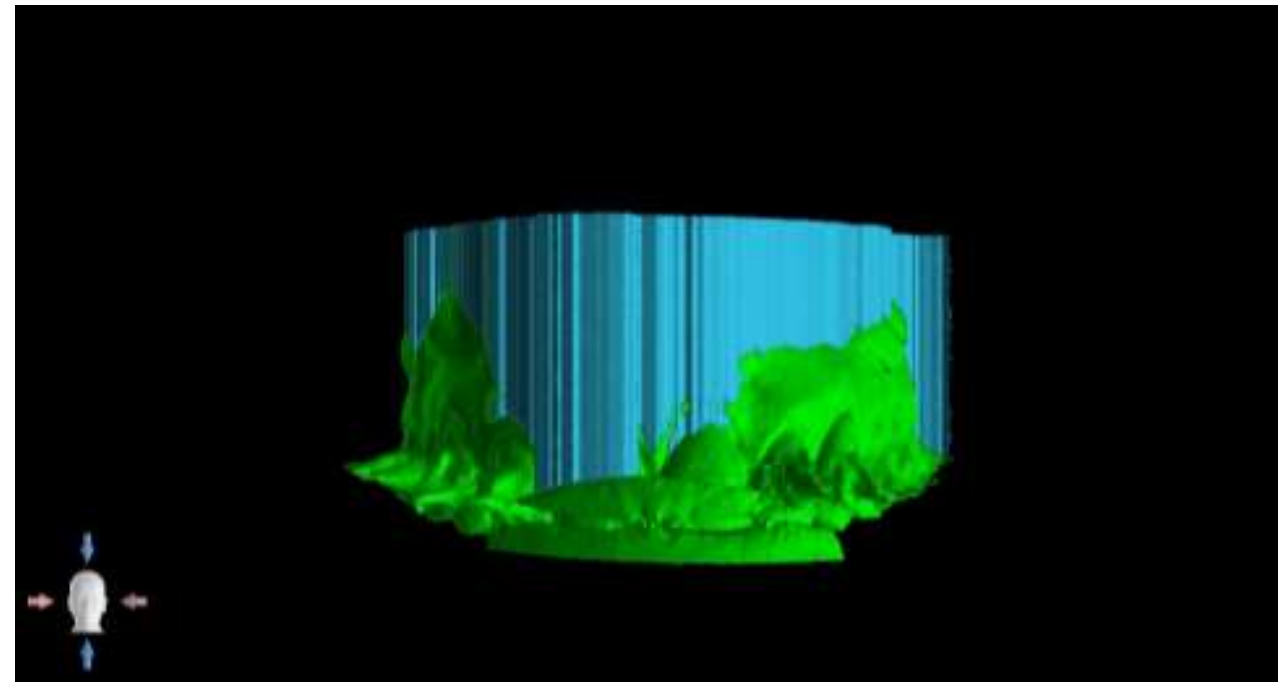

Fonte: Autores.

Figura 7. Resultado do vazamento digital, no qual pode-se observar características fidedignas da superfície da mucosa do paciente agora de maneira digitalizada e propícia para a manipulação digital.

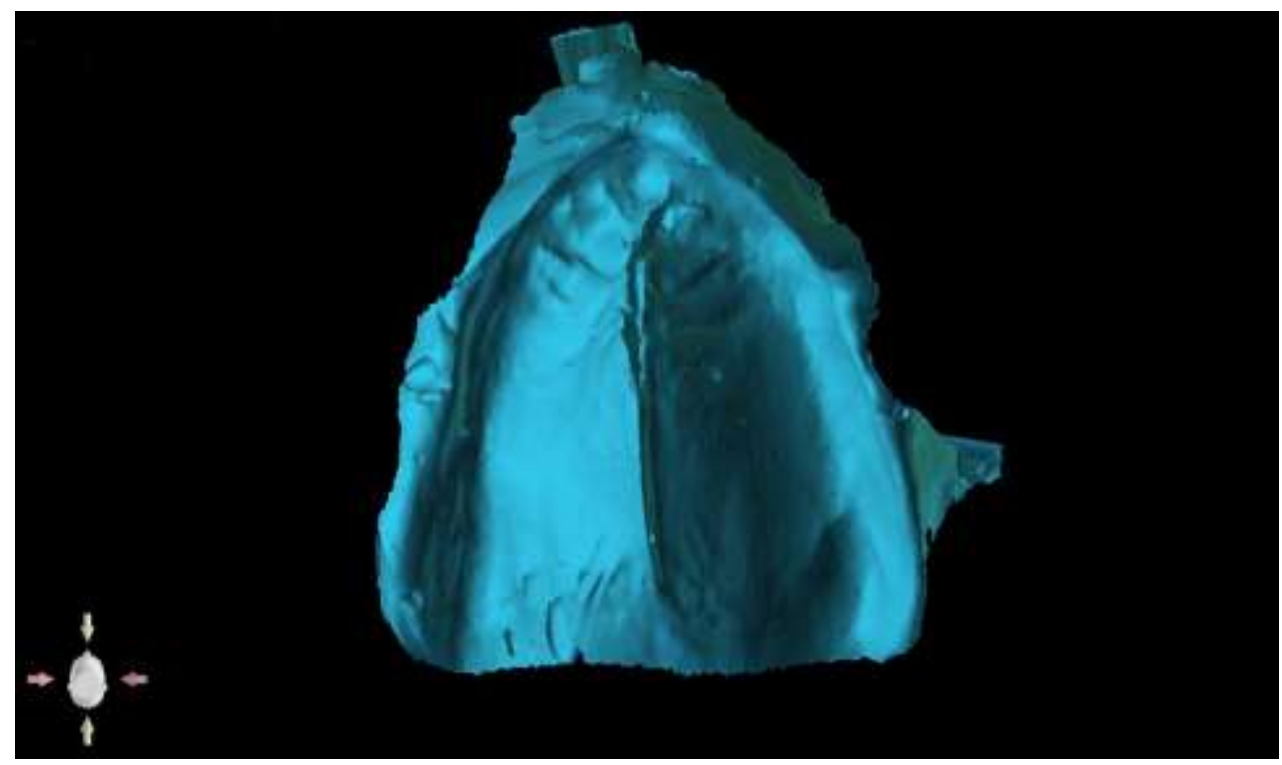

Fonte: Autores.

Após a fusão da imagem, iniciou-se o planejamento cirúrgico de implante conduzido de modo virtual, usando software de planejamento de implante, podendo projetar por meio digital o diâmetro, o comprimento e a posição 3D dos implantes (Figura 8). Esse método leva em consideração todos os parâmetros estabelecidos em cirurgias de instalação de implantes de forma convencional, respeitando as dimensões dos implantes, a qualidade óssea, a proximidade desses com estruturas nobre e a outros implantes (Mizutani et al., 2020). 
Figura 8. Simulação digital de instalação e posicionamento dos implantes por meio de software, no qual observa-se o plano de inclinação dos implantes em relação a arcada superior do paciente, tendo como referência as imagens STL e DICOM.

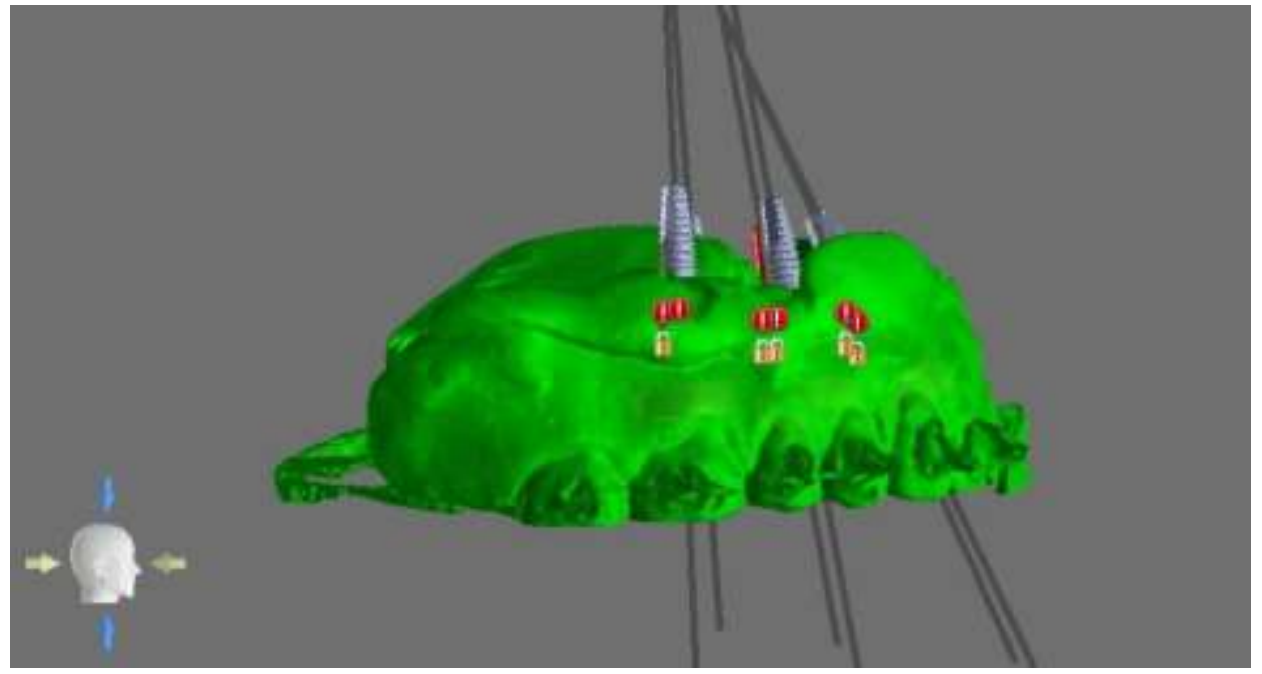

Fonte: Autores.

Nessa fase de planejamento, adicionou-se à guia cirúrgica as anilhas virtuais, que serão produzidas em titânio e instaladas no guia (Figura 9). Essas anilhas agem como limitadores de profundidade e guiam a angulação de instalação dos implantes. Adicionou-se também três orifícios no guia, onde foram instalados os pinos de fixação, que agem sobre pressão. Esses pinos servem para manter o guia cirúrgico no local correto da cavidade bucal e evitar assim erros no processo de osteotomia, em casos de movimentação indesejada do guia.

Figura 9. Adição virtual de anilhas limitadoras de profundidade demonstradas em laranja, posicionadas por meio de software em locais onde serão instalados os implantes, tendo como base o modelo virtual, em azul.

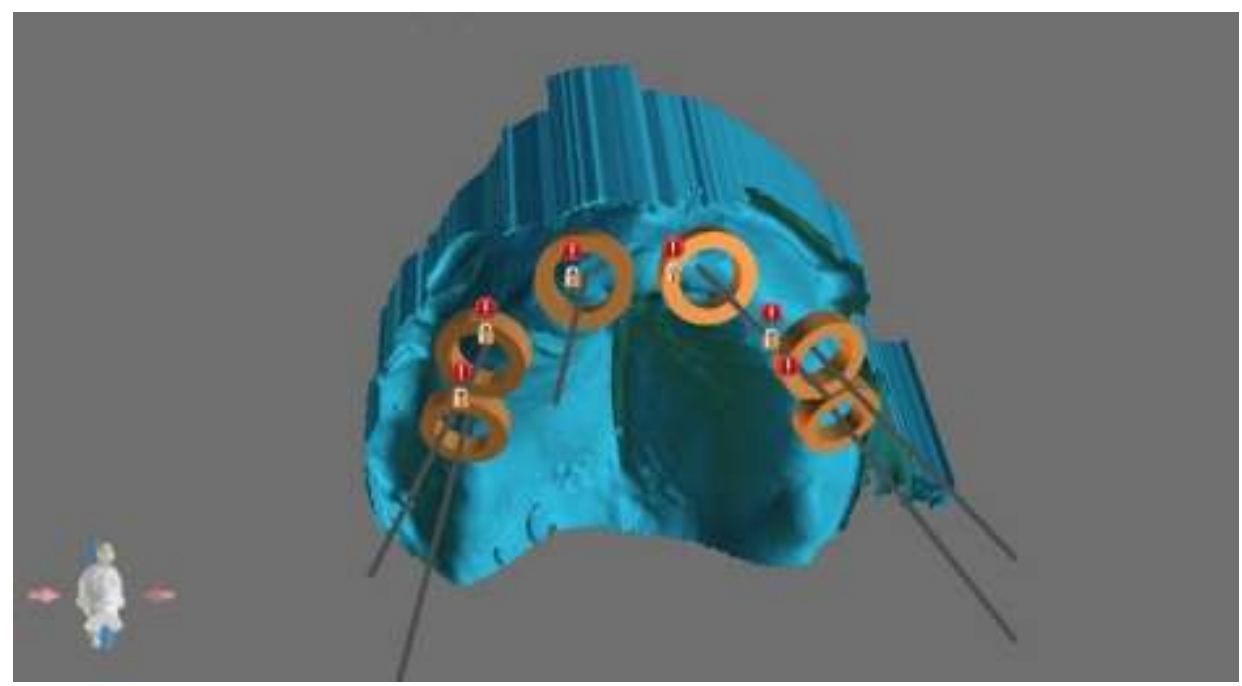

Fonte: Autores.

Assim que o planejamento dos implantes foi concluído, os dados foram transferidos por meio de arquivos em CAD/CAM para uma impressora 3D (Photon, Anycubic, China) que utiliza como material do guia cirúrgico uma resina biocompatível (Cosmos SG, Yller, Brasil). Esse aparelho de estereolitografia usa um laser de varredura para construir os guias cirúrgicos, depositando uma camada por vez, em um tanque de resina fotopolimérica fotopolimerizável. As camadas são feitas pelo laser na superfície da resina líquida. Assim, a plataforma de construção do aparelho desce e passa outra camada de resina, 
que é depositada na superfície, repetindo assim esse processo até o término da construção da peça (Dawood et al., 2015) (Figura 10).

Figura 10. Guia cirúrgico impresso por impressora 3D, com utilização de resina biocompatível, o qual pode-se observar as anilhas para perfuração e pontos para a inserção dos pinos de fixação.

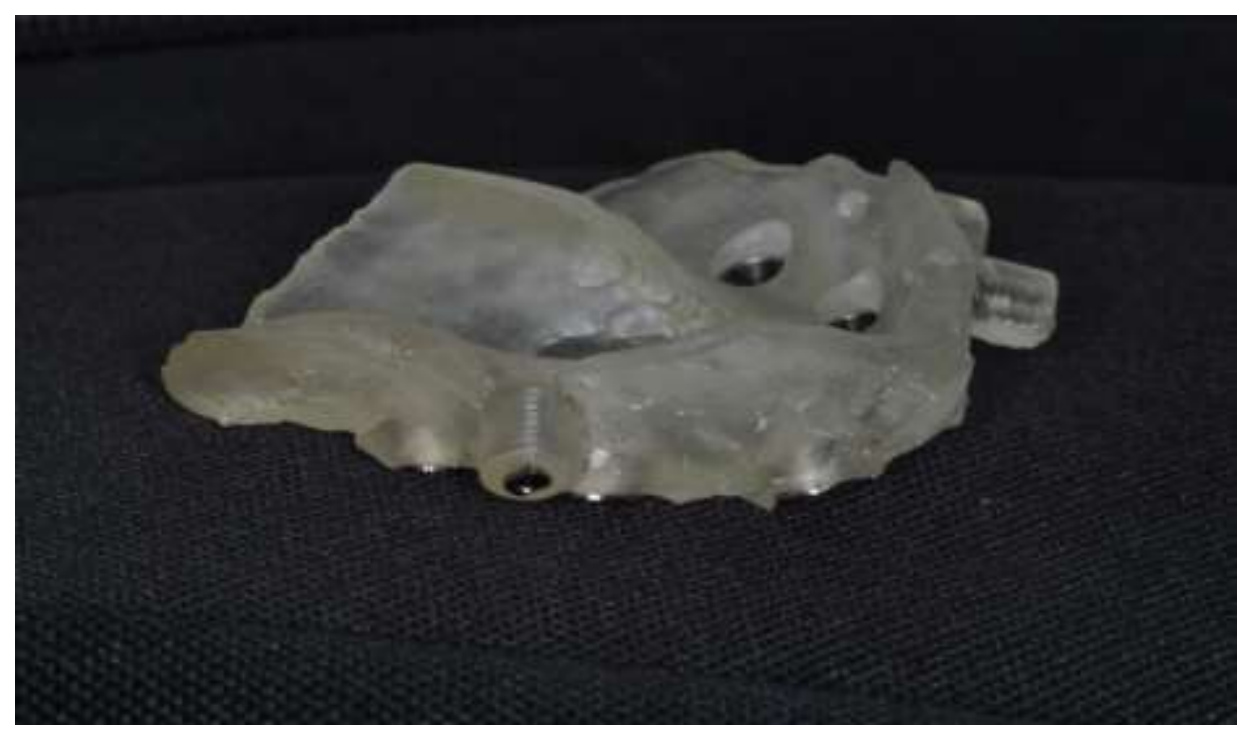

Fonte: Autores.

Após o processo de construção do guia, fez-se um teste pré-cirúrgico da peça para checar sua adaptação. Visto que se encaixou corretamente, iniciou o protocolo cirúrgico. A cirurgia de implante sem retalho guiada por computador, foi realizada sob anestesia local, na arcada superior. O guia cirúrgico foi encaixado na cavidade oral do paciente e fixado no lugar correto com 3 (três) pinos de estabilização (Figura 11). Seguindo os protocolos cirúrgicos preconizados no kit de fresagem, foram usados 6 (seis) implantes do modelo hexágono externo de 4.1x3.75, de 13mm, usando o sistema STRONG SW da S.I.N Implant System (SIN implantes, São Paulo/SP) (Figura 12).

Figura 11. Adaptação intra-oral do guia para perfuração. Observar o posicionamento das anilhas e pinos de fixação, além da acomodação da peça em relação às estruturas anatômicas do paciente.

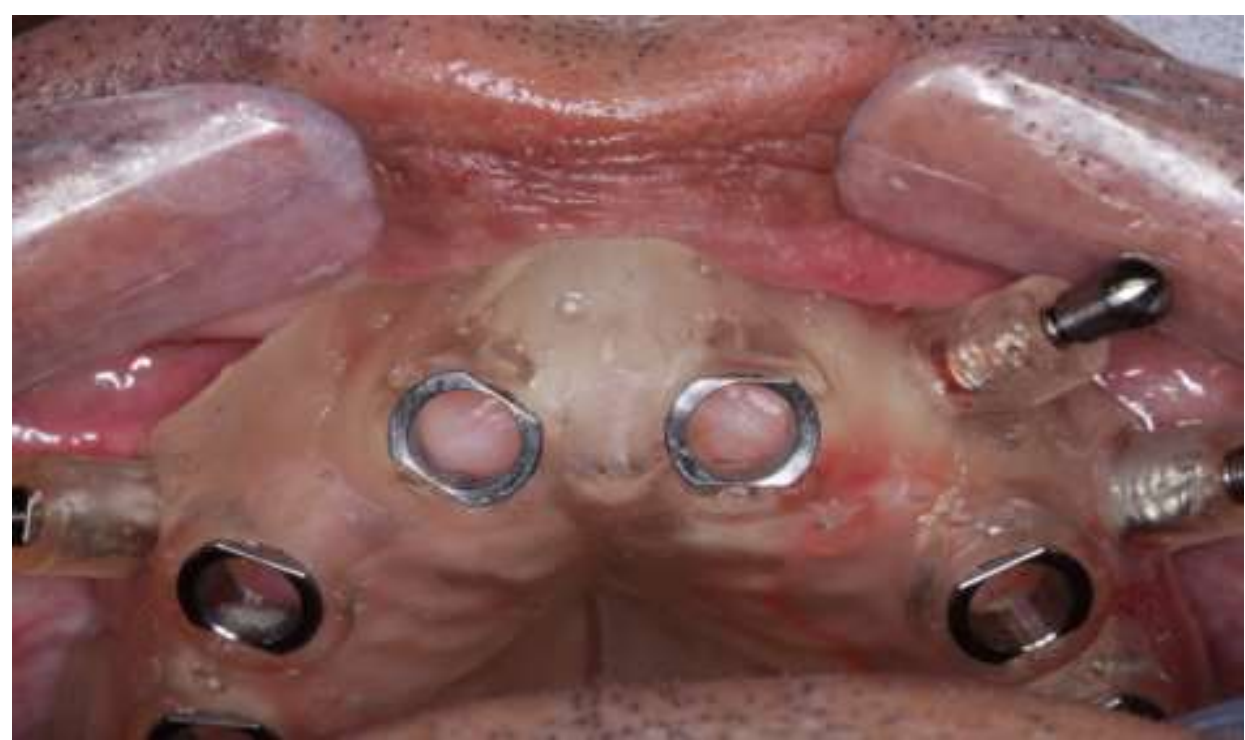

Fonte: Autores. 
Figura 12. Pós-operatório imediato, com 6 implantes instalados. Observar a manutenção de tecido e as mínimas incisões necessárias para se instalar os implantes. Observar também a distribuição em formato hexagonal dos implantes na arcada superior, o que auxilia na dissipação correta de cargas oclusais sobre os implantes.

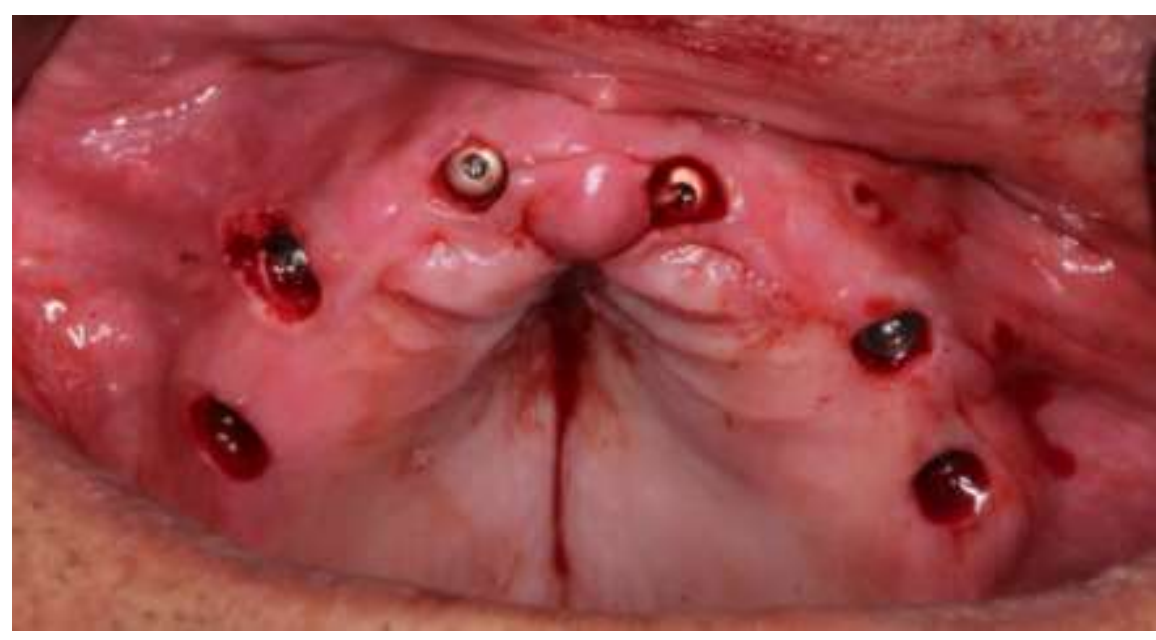

Fonte: Autores.

\section{Resultados}

Seguindo todos os parâmetros estabelecidos no planejamento digital, a cirurgia foi rápida e sem obstáculos, seguiu todos os passos necessários de fresagem sem maiores complicações. Nenhum implante foi perdido, o paciente apresentou poucas dores e edema nos dias posteriores à cirurgia. Durante a cicatrização pós-operatória, os implantes alcançaram o sucesso na osseointegração e a cicatrização ocorreu sem intercorrências, ou seja, sem patologias sinusais, lesões nervosas, reações alérgicas e infecções. Os exames radiológicos e clínicos mostraram estabilidade adequada do implante e boa cicatrização dos tecidos moles durante o primeiro retorno, que foi após 30 dias. o paciente recebeu a prótese total provisória de acordo com a planejamento inicial (Figura 13).

Figura 13. Paciente com a prótese total superior provisória. Observar as proporções faciais devolvidas e os parâmetros estéticos e oclusais reestabelecidos.

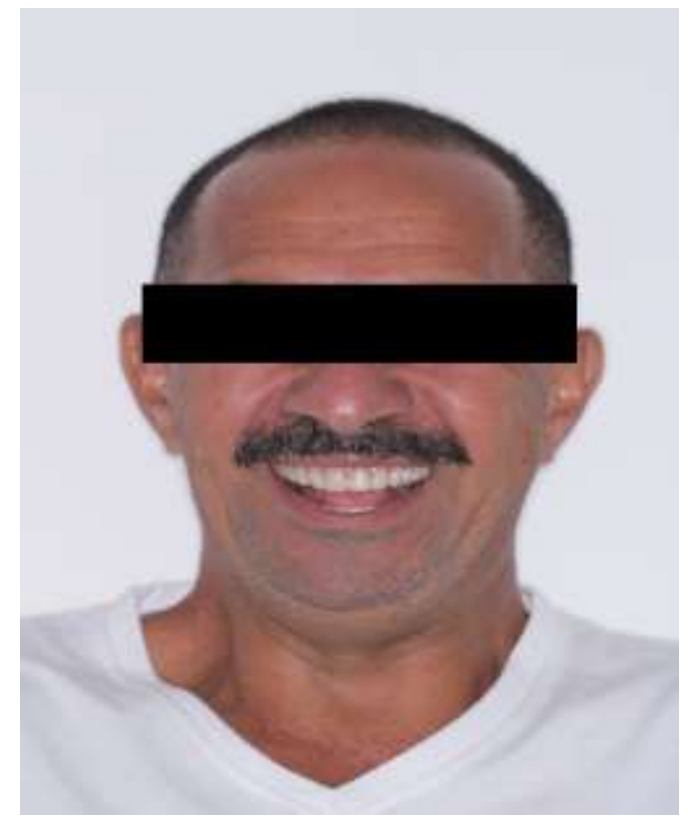

Fonte: Autores. 
Levando-se em consideração o tamanho da amostra inscrita, não foi possível realizar uma análise estatística no presente estudo preliminar, entretanto, os dados clínicos e radiológicos obtidos nesse caso supõe que o planejamento digital para cirurgia de implante guiada por computador foi satisfatório, assim como a confecção do guia cirúrgico, a prótese provisória e o processo cirúrgico.

\section{Discussão}

O objetivo deste relato de caso foi expor o fluxo de planejamento digital de uma cirurgia de colocação de implantes sem retalho guiada por computador em um paciente classificado como Classe III de Angle, mordida cruzada anterior e periodontite propriamente dita, estágio IV, grau C, generalizada. No qual fez-se extração de todos os dentes comprometidos e reabilitação protética com prótese total sobre implante.

As novas tecnologias digitais têm trazido um benefício enorme para os tratamentos odontológicos modernos. O desenvolvimento e evolução de CBCT e scanners intraorais, que são utilizados em consultórios odontológicos, proporcionaram aos cirurgiões-dentistas poderosas ferramentas de imagem e recursos em softwares. Assim, a cirurgia de implante guiada por computador ganhou popularidade na odontologia devido aos avanços do uso de tomografia computadorizada de feixe cônico (CBCT) e planejamento do tratamento de implantes com utilização de softwares que avaliam a localização ideal do implante (Garib et al., 2007).

O planejamento pré-operatório da posição do implante é uma etapa importante da coordenação cirúrgica e protética para alcançar resultados funcionais e estéticos satisfatórios. A CBCT é um método seguro, não invasivo e acessível para descrever as estruturas maxilofaciais avaliando a quantidade e a qualidade do osso das cristas alveolares. Assim, permite a utilização de marcadores fiduciais para relacionar os dados da TC com o plano protético (Greenberg, 2015).

Segundo An et al., 2019, no método de registro utilizado nesse caso, a prótese total provisória deve estar bem adaptada para que se possa obter uma impressão maxilar com marcadores de resina satisfatória. No momento da varredura, o paciente precisa estar em oclusão para que a mucosa seja comprimida pela prótese e assim registrar de forma fidedigna a mucosa. Esse processo ocorre com o objetivo de evitar espaços entre a mucosa do paciente e a prótese, que pode ocasionar em imprecisões na fabricação do guia cirúrgico, pois esse guia será fabricado com base na prótese digitalizada.

Segundo Bernardo., 2015, o objetivo principal dessa técnica é aprimorar a precisão protética, de diagnóstico e cirúrgica, tendo uma abordagem mais rápida e simplificada. Este novo conceito é visto na literatura como previsível, seguro e entrega menos desconforto durante o período de cicatrização, visto que não há a necessidade de abrir retalho, o que gera uma redução no tempo cirúrgico. Além disso, tem como vantagem a preservação do tecido mole e do volume de tecido duro no sítio cirúrgico, podendo integrar os determinantes restauradores no planejamento cirúrgico, gerando um resultado protético mais estético, funcional e previsível. (Beretta et al., 2014).

Em contra partida, Nikzad e Azari, 2008, demonstra algumas desvantagens do planejamento digital para cirurgias guiada. Os pacientes são mais expostos à radiação, devido às tomadas tomográficas, que tem doses mais elevadas do que as técnicas radiográficas. A interpretação das imagens tomográficas é difícil, a transferências de informações e dados são laboriosas, gerando a necessidade do uso de softwares de processamento de imagens, exigindo assim um treinamento prévio do profissional. Além disso, essa técnica tem um maior custo para o paciente.

A cirurgia guiada torna-se mais benéfica quando há a necessidade de instalação de três ou mais implantes, quando há proximidade com alguma estrutura anatômica vital, quando se tem um volume ósseo questionável (largura e altura deficiente), para pacientes com debilidades físicas, psicológicas e médicas ou para pacientes que têm alguma alteração significativa de tecidos, por trauma ou cirurgia prévia (Orentlicher et al., 2012). 
Nesse caso relatado, fez-se a escolha por essa técnica devido a posição crítica em que os implantes seriam instalados, visto que o paciente apresentava uma mordida cruzada anterior. Dessa forma, o planejamento digital pôde prever qual o posicionamento de instalação dos implantes mais satisfatório, o eixo de distribuição de força mastigatórias entre esses implantes e quais parâmetros estéticos mais adequados, afim de diminuir essa deficiência oclusal.

Segundo Hultin et al., 2012, se comercializa um conceito da cirurgia de implantes guiada por computador com carga imediata, como sendo uma abordagem segura, fácil e previsível. Entretanto, o autor exclama que essa técnica também estar sujeita a complicações e falhas, principalmente no momento da colocação guiada dos implantes. A complicação cirúrgica mais comum é a fratura do guia cirúrgico e a complicação protética mais comum é a não adaptação da prótese, esse tipo de falha não ocorre ou não foram relatados no método cirúrgico convencional.

Schneider et al., 2009, relata em seu estudo que podem ocorrer outras complicações relacionadas ao planejamento digital incorreto, como erro na tomada tomográfica, no processo de varredura do scanner intraoral, erros do software de planejamento, na prototipagem do guia que são erros intrínsecos à impressora 3D, que pode estar descalibrada e também na transferência de informações. Já D'haese et al., 2010, reporta como complicações o afrouxamento da prótese, mordidas bilaterias das bochechas, defeitos na fala e principalmente sobreaquecimento ósseo no momento da osteotomia, devido à dificuldade de irrigação com substância salina da superfície óssea, pois o guia pode cobrir do campo operatório.

Ademais, a técnica utilizada nesse caso é uma ferramenta enriquecedora para alcançar resultados bem-sucedidos, visto que o uso clínico de cirurgias de implante guiada por computados permite resultados mais favoráveis e previsíveis em termos de função, conforto e estética. Esse método digital entrega tratamentos individualizados e personalizados para os pacientes e agregam de forma substancial em casos que o método convencional poderia encontrar maiores chances de intercorrências (Pinto, 2017).

\section{Conclusão}

$\mathrm{Na}$ técnica de cirurgia guiada assistida por computador, com dupla varredura, com imagens obtidas por CBCT, ocorre o processamento por software que permite planejamento da instalação ideal de implantes de forma virtual. As tecnologias digitais utilizadas nessa técnica odontológica permitem uma avaliação e seleção de locais cirúrgicos dos implantes em um modelo virtual, tendo como referência as características anatômicas da maxila e mandíbula edêntula, estabelecendo assim um protocolo clínico, cirúrgico mais favorável à reabilitação funcional e estética do paciente. Para que se tenha sucesso no processo, uma anamnese detalhada, seguida de uma avaliação e indicação correta é se suma importância, além de um bom planejamento e execução da técnica pelo profissional.

Portanto, sugere-se que as técnicas de planejamento digital para instalação de implantes por meio de guias cirúrgicos impressos por impressoras 3D descritas anteriormente, são de grande valia para o processo clínico, entretanto novos estudos controlados e randomizados podem ser escritos, afim de demonstrar a eficácia, a eficiência e o desempenho clínico em curto, médio e longo prazo dessa técnica.

\section{Referências}

Alvares, H. L. P. (2020) Planejamento odontológico virtual aplicado para cirurgia guiada de implante unitário: relato de caso. (monografia). Centro Universitário - UNDB, São Luís, Brasil.

Arcuri, L., Lorenzi, C., Cecchetti, F., Germano, F., Spuntarelli, M., \& Barlattani, A. (2016). Full digital workflow for implant-prosthetic rehabilitations: a case report. ORAL \& implantology, 8(4), 114-121. https://doi.org/10.11138/orl/2015.8.4.114.

Beretta, M., Poli, P. P., \& Maiorana, C. (2014). Accuracy of computer-aided template-guided oral implant placement: a prospective clinical study. Journal of Periodontal \& Implant Science, 44(4), 184. https://doi.org/10.5051/jpis.2014.44.4.184. 
Bernardo, R. M. P. C. (2015) Cirurgia guiada na colocação de implantes. (monografia). Faculdade de Medicina Dentária da Universidade do Porto, Porto, Portugal.

Caton, J. G. et al. (1999) A new classification scheme for periodontal and peri-implant diseases and conditions-introduction and key changes from the 1999 classification. 2018. Journal of periodontology, 89(1), 1-8. Recuperado em: https://aap.onlinelibrary.wiley.com/doi/epdf/10.1002/jper.18-0157.

Cunha, R. M., Souza, F. Á., Hadad, H., Poli, P. P., Maiorana, C., \& Carvalho, P. S. P. (2020a). Accuracy evaluation of computer-guided implant surgery associated with prototyped surgical guides. The Journal of Prosthetic Dentistry. https://doi.org/10.1016/j.prosdent.2019.07.010.

Dawood, A., Marti, B. M., Sauret-Jackson, V., \& Darwood, A. (2015b). 3D printing in dentistry. British Dental Journal, 219(11), 521529. https://doi.org/10.1038/sj.bdj.2015.914.

D'haese, J., Van De Velde, T., Komiyama, A., Hultin, M., \& De Bruyn, H. (2010). Accuracy and Complications Using Computer-Designed Stereolithographic Surgical Guides for Oral Rehabilitation by Means of Dental Implants: A Review of the Literature. Clinical Implant Dentistry and Related Research, 14(3), 321-335. https://doi.org/10.1111/j.1708-8208.2010.00275.x.

Garib, D. G., Raymundo Jr., R., Raymundo, M. V., Raymundo, D. V., \& Ferreira, S. N. (2007). Tomografia computadorizada de feixe cônico (Cone beam): entendendo este novo método de diagnóstico por imagem com promissora aplicabilidade na Ortodontia. Revista Dental Press de Ortodontia e Ortopedia Facial, 12(2), 139-156. https://doi.org/10.1590/s1415-54192007000200018.

Hultin, M., Svensson, K. G., \& Trulsson, M. (2012). Clinical advantages of computer-guided implant placement: a systematic review. Clinical Oral Implants Research, 23, 124-135. https://doi.org/10.1111/j.1600-0501.2012.02545.x.

Jain, S., Choudhary, K., Nagi, R., Shukla, S., Kaur, N., \& Grover, D. (2019). New evolution of cone-beam computed tomography in dentistry: Combining digital technologies. Imaging Science in Dentistry, 49(3), 179. https://doi.org/10.5624/isd.2019.49.3.179.

Klokkevold, P. (2007) Implantodontia oral. in M. G, N. Carranza (ED. 11), Periodontia clínica. (PP 825-840) Elsevier Brasil.

Kraft, B. (2019) Avaliação da Influência da Cirurgia Guiada na Precisão do Posicionamento de Implantes Unitários em Alvéolos na Região Anterior-Estudo Clínico Randomizado. (Dissertação de mestrado). Instituto Latino Americano de Pesquisa e Ensino Odontológico - ILAPEO, Curitiba, Brasil.

Mizutani, F. S; Araújo, F. M; Rodrigues, T. S; Machado, V. C. (2020) Fluxo de trabalho digital para cirurgia de implante unitário e reabilitação protética em área estética - relato de caso. Revista Full Dentistry in science, 11(42), 11-19. https://editoraplena.com.br/produto/colunainformacao-e-tecnologia-fluxo-de-trabalho-digital-para-cirurgia-de-implante-unitario-e-reabilitacao-protetica-em-area-estetica-relato-de-caso.

Nikzad, S., \& Azari, A. (2008). Computer-assisted implant surgery; a flapless surgical/immediate loaded approach with 1 year follow-up. The International Journal of Medical Robotics and Computer Assisted Surgery, 4(4), 348-354. https://doi.org/10.1002/rcs.219.

Orentlicher, G., Goldsmith, D., \& Abboud, M. (2012). Computer-Guided Planning and Placement of Dental Implants. Atlas of the Oral and Maxillofacial Surgery Clinics, 20(1), 53-79. https://doi.org/10.1016/j.cxom.2011.12.004.

Pereira, R. A; Siqueira, L. S; Romeiro, R. L. (2019) Cirurgia guiada em implantodontia: relato de caso. Revista Ciência e Saúde On-line, 4(1), 34-42. Recuperado em: https://revistaeletronicafunvic.org/index.php/c14ffd10/article/view/135.

Pereira, A. S., et al. (2018). Metodologia da pesquisa científica UFSM. https://repositorio.ufsm.br/bitstream/handle/1/15824/Lic_Computacao_MetodologiaPesquisa-Cientifica.pdf?sequence $=1$.

Pinto, A. (2017). Postextraction computer-guided implant surgery in partially edentate patients with metal restorations: a case report. Oral \& Implantology, 10(1), 71. https://doi.org/10.11138/orl/2017.10.1.071.

Schneider, D., Marquardt, P., Zwahlen, M., \& Jung, R. E. (2009). A systematic review on the accuracy and the clinical outcome of computer-guided templatebased implant dentistry. Clinical Oral Implants Research, 20, 73-86. https://doi.org/10.1111/j.1600-0501.2009.01788.x.

Veríssimo, A. H., Souza, J. A. N. de Oliveira, T. A., Gonçalves, A. G., Afonso, F. A. C., \& Souza Júnior, F. A. (2021). Oral rehabilitation with dental implant and immediate loading by guided surgery: case report. Research, Society and Development, 10(1), e4810110854. https://doi.org/10.33448/rsd-v10i1.10854. 